\title{
A human-mouse conserved sex bias in amygdala gene expression related to circadian clock and energy metabolism
}

\author{
Li-Chun Lin, David A Lewis and Etienne Sibille*
}

\begin{abstract}
Background: Major depression affects twice as many women as men, but the underlying molecular mechanisms responsible for the heightened female vulnerability are not known. The amygdala, composed of heterogeneous subnuclei, participates in multiple functional circuits regulating emotional responses to stress. We hypothesized that sex differences in molecular structure may contribute to differential mood regulation and disease vulnerability.

Findings: Using gene arrays followed by quantitative PCR validation, we compared the transcriptome profiles between sexes in human and mouse amygdala. We now report sexually dimorphic features of transcriptomes in the basolateral nucleus of the amygdala, and these features are highly conserved across species. A functional analysis of differential gene expression showed that mitochondrial-related gene groups were identified as the top biological pathways associated with sexual dimorphism in both species.

Conclusions: These results suggest that the basolateral amygdala is a sexually dimorphic structure, featuring a regulatory cascade of mitochondrial function and circadian rhythm, potentially linked through sirtuins and hormone nuclear receptors. Hence, baseline differences in amygdalar circadian regulation of cellular metabolism may contribute to sex-related differences in mood regulation and vulnerability to major depression.
\end{abstract}

\section{Introduction}

Males and females differ in behavior and brain structure, as well as in prevalence of neuropsychiatric disorders. The greater prevalence of major depression and rapid cycling bipolar disorder in women [1,2] highlights the importance of studying potential mechanisms for sex differences. Sex chromosome- and hormone-linked genes may directly affect sexually dimorphic neurobiology [3]. For example, sex hormones regulate the size of the medial amygdala and bed nucleus of the stria terminalis during development [4]. The lateral (LA) and basal (basolateral; throughout the article we refer to human BA and mouse BLA) nuclei of the amygdala have been identified as critical sites for learned fear and emotion regulation in healthy subjects and in patients with mood disorders [5,6], but little is known about their sexual dimorphism. The isolation of subnuclei from the

\footnotetext{
* Correspondence: sibilleel@upmc.edu Translational Neuroscience Program, Department of Psychiatry, and Center for Neuroscience, 3811 O'Hara Street, University of Pittsburgh, Pittsburgh, PA 15213, USA
}

(c) 2011 Lin et al; licensee BioMed Central Ltd. This is an Open Access article distributed under the terms of the Creative Commons Attribution License (http://creativecommons.org/licenses/by/2.0), which permits unrestricted use, distribution, and reproduction in any medium, provided the original work is properly cited.

\section{Methods}

\section{Human Brain Samples}

Postmortem amygdala samples from 12 healthy male controls and 12 healthy female controls (age = 39-64 years) were obtained from the University of Pittsburgh Brain Donation Program (Table 1), as previously described [6]. Male and female groups were matched in pairs as closely as possible on the basis of RNA integrity, $\mathrm{pH}$, post-mortem interval, age, and sex, and the groups did not differ on any of these parameters ( $p>0.05$; Table 1). All subjects died suddenly without prolonged

amygdala complex improves microarray sensitivity to detect differences in gene expression by reducing sample fication of genetic sex differences. To characterize sex differences in the intrinsic molecular properties of the dimgla, we examined the presence of sexually nuclei of the amygdala using postmortem samples from control human subjects and mice. 
Table 1 Characteristics of matched human subjects

\begin{tabular}{|c|c|c|c|c|c|c|c|c|c|c|c|c|}
\hline \multirow[b]{2}{*}{ Pair } & \multicolumn{6}{|c|}{ Female Group } & \multicolumn{6}{|c|}{ Male Group } \\
\hline & Case & $\begin{array}{c}\text { Age } \\
\text { (Years) }\end{array}$ & Race & $\begin{array}{l}\text { Interval } \\
\text { (Hours) }\end{array}$ & $\mathrm{pH}$ & RIN & Case & $\begin{array}{c}\text { Age } \\
\text { (Years) }\end{array}$ & Race & $\begin{array}{l}\text { Interval } \\
\text { (Hours) }\end{array}$ & $\mathrm{pH}$ & RIN \\
\hline 1 & HU1282 & 39 & W & 24.5 & 6.8 & 7.5 & HU604 & 39 & W & 19.3 & 7.1 & 8.6 \\
\hline 2 & HU1092 & 40 & B & 16.6 & 6.8 & 8.0 & HU1047 & 43 & W & 12.0 & 6.6 & 9.0 \\
\hline 3 & HU627 $7^{a, b}$ & 43 & B & 14.1 & 7.1 & 7.0 & $\mathrm{H} \cup 857^{\mathrm{a}, \mathrm{b}}$ & 48 & W & 16.6 & 6.7 & 8.9 \\
\hline 4 & HU567 & 46 & W & 15.0 & 6.8 & 8.9 & $\mathrm{HU} 1067^{\mathrm{b}}$ & 49 & W & 6.0 & 6.6 & 8.2 \\
\hline 5 & $\mathrm{HU} 1280^{\mathrm{a}}$ & 50 & W & 23.5 & 6.7 & 7.7 & HU1086 $6^{a, b}$ & 51 & W & 24.2 & 6.8 & 8.1 \\
\hline 6 & HU1391 a,b & 51 & W & 7.8 & 6.6 & 7.1 & HU852 $2^{a, b}$ & 54 & W & 8.0 & 6.8 & 9.1 \\
\hline 7 & HU686 & 52 & W & 22.6 & 7.1 & 8.5 & HU1031 ${ }^{b}$ & 53 & W & 23.2 & 6.8 & 8.9 \\
\hline 8 & HU575 & 55 & B & 11.3 & 6.8 & 9.6 & HU1122 & 55 & W & 15.4 & 6.7 & 7.9 \\
\hline 9 & HU1247 & 58 & W & 22.7 & 6.4 & 8.4 & HU685 & 56 & W & 14.0 & 6.6 & 8.0 \\
\hline 10 & HU1318 & 58 & W & 18.8 & 6.7 & 7.4 & HU852 & 54 & W & 8.0 & 6.8 & 9.1 \\
\hline 11 & HU568 & 60 & W & 9.5 & 6.9 & 8.7 & HU551 & 61 & W & 16.4 & 6.6 & 8.3 \\
\hline 12 & HU1466 ${ }^{\mathrm{b}}$ & 64 & B & 20.0 & 6.7 & 8.8 & HU615 & 62 & W & 7.2 & 6.4 & 7.8 \\
\hline \multicolumn{13}{|c|}{ Matched samples processed for microarray: } \\
\hline & AVG & 51.3 & & 17.2 & 6.8 & 8.1 & & 52.1 & & 14.2 & 6.7 & 8.5 \\
\hline & STDEV & 8.1 & & 5.7 & 0.2 & 0.8 & & 6.7 & & 6.2 & 0.2 & 0.5 \\
\hline \multicolumn{13}{|c|}{ Matched LA for final array analysis: } \\
\hline & AVG & 52.4 & & 17.9 & 6.8 & 8.4 & & 52.4 & & 13.5 & 6.7 & 8.4 \\
\hline & STDEV & 8.9 & & 5.2 & 0.2 & 0.7 & & 7.7 & & 5.8 & 0.2 & 0.5 \\
\hline \multicolumn{13}{|c|}{ Matched BA for final array analysis: } \\
\hline & AVG & 50.9 & & 18.3 & 6.8 & 8.3 & & 52.9 & & 13.2 & 6.7 & 8.4 \\
\hline & STDEV & 7.8 & & 5.5 & 0.2 & 0.7 & & 8.7 & & 4.4 & 0.2 & 0.5 \\
\hline
\end{tabular}

Samples from LA (marked ${ }^{\mathrm{a}}$ ) and BA (marked ${ }^{\mathrm{b}}$ ) processed but excluded due to lower microarray quality control; W = Caucasian; B = African American; RIN = RNA integrity number; AVG = average; STDVE = standard deviation.

agonal periods. See [6] for additional details. In contrast to the light acetylcholinesterase (AChE)-stained LA across species, human BA and mouse BLA contained the heavy stained AChE-positive neuropil in the amygdala $[7,8]$, implying that human BA and LA anatomically correspond to the mouse BLA and LA according to the well-defined AChE boundaries. Hence, based on the distribution of AChE activity and thionin staining, human lateral (LA) and basal (BA) amygdala nuclei were defined and separately harvested from $20-\mu \mathrm{m}$ cryostat sections using a clean RNase-free pipette tip. All procedures were approved by the University of Pittsburgh's Committee for the Oversight of Research Involving the Dead and Institutional Review Board for Biomedical Research.

\section{Mouse Brain Samples}

Brains from adult C57BL/6J mice ( $n=5$ per sex; pooled by 2 from 10 mice/group, 3 months of age) were harvested, fresh frozen and cryo-sectioned at $20 \mu \mathrm{m}$ on UV-treated laser-capture microscopy slides (Leica Microsystems, Wetzlar, Germany). Sections on the slides were dried and stored at $-80^{\circ} \mathrm{C}$ until used. Lateral (LA) to basolateral (BLA) amygdala nuclei were visualized with rapid thionin staining and dissected with the Leica
LMD 6500 laser microdissection system. All mice were maintained on a 12-hour light-dark cycle (lights on 06:00 and lights off on 18:00) with ad libitum food and water. All procedures were approved by the University of Pittsburgh Institutional Animal Care and Use Committee (protocol \# 0911014A-2, Animal Assurance \# A3187-01).

\section{RNA extraction for Microarray Samples}

Total RNA was extracted using the RNeasy Plus Micro Kit (QIAGEN, Valencia, CA) and processed for microarray analysis according to manufacturer's protocol. RNA samples were amplified with Illumina TotalPrep RNA Amplification Kit (for human tissue; Illumina, San Diego, CA) and Nugen WT-Ovation Pico RNA Amplification System (for mouse tissue; NuGen, San Carlos, CA). The fragmented labeled cRNA samples were processed and hybridized to microarrays (Human HT-12v3 and MouseWG-6v2.0 BeadChips).

\section{Array Data Analysis}

For statistical analysis, the $\log _{2}$-transformed signal intensities of probe-sets that passed the initial expression filters (<30\% missing values, detection $\mathrm{p}$-value $>0.1$ ), were extracted and renormalized to the same mean and 
variance across arrays in order to control for any effects of batch. Discovery threshold for male/female differences were applied in both species (two-tailed $t$-test $p$ value $<0.05$; $>20 \%$ effect size). Functional analyses were performed on the identified gene list using the Ingenuity Pathways Knowledge Base [9] and compared across species.

\section{Real-time Quantitative PCR (qPCR) Data Analysis}

Separate RNA samples were reverse-transcribed using QScript cDNA super mix (QuantaBioscience, Gaithersburg, MD) using a combination of oligo (dT) and random primers. Each PCR reaction was performed in triplicate and compared to three internal controls (GAPDH, beta-actin and cyclophilin). All designed primer sequences were designed with Primer3Plus (Additional File 1, table S1). Results were calculated as the geometric mean of relative intensities compared to the three internal controls. Validation of the array results was considered at $p<0.05$, using one-tailed unpaired $t$-test.

\section{Results}

Using exploratory criteria we generated large and informative parallel human/mouse transcriptome datasets from male and female BA (BLA in mice) and LA nuclei of the amygdala. As an internal control to assess the sensitivity of the array approach, all detected Y-chromosome genes (male-specific) displayed significantly malebiased expression, and expression of numerous X-linked genes showed a female bias (Figure 1). In humans, 1335 sex-biased autosomal genes were identified in BA (Additional File 2, table S2), and fewer $(n=165)$ in LA (Additional File 3, table S3). In mice, $\sim 1 \%$ of autosomal genes displayed sex-biased expression (BLA, $n=515$, Additional File 4, table S4; LA, $n=556$, Additional File 5, table S5).

To reduce false discovery and focus on the most relevant changes, we characterized the functional and crossspecies relatedness of autosomal sex-biased gene sets using the Ingenuity Pathways Analysis (IPA) database. Mitochondrial-related functions were consistently identified among the top four most-represented pathways in human BA $\left(p<1.57 \mathrm{E}^{-06}\right)$, mouse BA $\left(p<8.37 \mathrm{E}^{-03}\right)$ and mouse LA $\left(p<6.72 \mathrm{E}^{-02}\right)$. Assembly of RNA Polymerase III Complex was the only identified canonical pathway in human LA and was at trend level $(p<0.1)$.

Within the mitochondrial-related functions, genes encoding enzymes of the mitochondrial electron transport chain (ETC) consistently displayed high male-biased expression (Figure 2A). Also, two genes coding for mitochondria-localized sirtuins (SIRT3, SIRT5), which serve as metabolic regulators of circadian rhythms by utilizing $\mathrm{NAD}^{+}[10]$, also showed similar high male-biased expression in human BA and mouse BLA. Of related interest was the identification of sex-biased circadian rhythm signaling in human BA (IPA, $p<5.39 \mathrm{E}^{-04}$ ) and mouse LA $\left(p<3.54 \mathrm{E}^{-02}\right)$, which included conserved high femalebiased expression of selected circadian clock genes (e.g., Period 2 and trend-level for CLOCK; Figure 2A). Intriguingly, as nuclear receptors link the circadian clock to metabolism [11], retinoic acid orphan receptor $\beta$ (RORB) and thyroid hormone receptor $\alpha$ (THRA) displayed high female-biased expression in human BA (RORB: average $\log$ ratio $[\mathrm{alr}]=0.29, p<0.026$; THRA: alr $=0.31$, $p<0.013)$.

The accuracy and reproducibility of the approach was assessed by qPCR on independent samples. 12 genes out of 18 tested were individually confirmed $(p<0.05$; same direction; Figure $2 \mathrm{~B}$ ). Overall, the $\mathrm{qPCR}$ validation results were highly correlated with array data using Pearson tests for significance $\left(r=0.86, p<2.5 \mathrm{E}^{-06}\right.$; Figure 2D).

We next evaluated the anatomical extent of the observed sex-biased gene profiles in other brain areas using existing human array data in cortical regions [12]. Numerous ETC and SIRT genes in dorsolateral prefrontal cortex (Brodman area 9 [BA9]; Figure 2C), and fewer in the ventrolateral prefrontal cortex (Brodman area 47 [BA47]; Figure 2C) and anterior cingulate cortex (ACC; Figure 2C), consistently displayed high male-biased expression, suggesting variable levels of regional conservation and amygdala enrichment.

Notably, within the limited cohort size and age range, we did not observe statistically significant age-related effects for genes confirmed by qPCR, except for malebiased ATP synthase genes (ATP5A1, $r=-0.78, p<$ 0.005; ATP6VOD1, $r=-0.58, p<0.05)$ in human male

\begin{tabular}{|c|c|c|c|c|c|c|c|c|}
\hline \multicolumn{9}{|c|}{ Sex Chromosome Genes } \\
\hline \multirow{4}{*}{$\begin{array}{l}\text { (number of genes) } \\
\text { Y-linked genes } \\
\text { X-linked genes }\end{array}$} & \multicolumn{4}{|c|}{ Human } & \multicolumn{4}{|c|}{ Mouse } \\
\hline & \multicolumn{2}{|c|}{ BA } & \multicolumn{2}{|c|}{ LA } & \multicolumn{2}{|c|}{ BLA } & \multicolumn{2}{|c|}{ LA } \\
\hline & $0 * / 13$ & $13 * / 13$ & $0 * / 13$ & $13 * / 13$ & $0 * / 5$ & $5 * / 5$ & $0 * / 6$ & $6 * / 6$ \\
\hline & $15^{*} / 48$ & $33 * / 48$ & $5 * / 12$ & $7 * / 12$ & $2 * / 3$ & $1 * / 3$ & $11 * / 26$ & $15^{*} / 26$ \\
\hline
\end{tabular}

Figure 1 Number of sex chromosome genes identified in the amygdala. Red: higher expressed in females; blue: lower expressed in females. In each cell, each gene in the numerator differed significantly in expression $(p<0.05)$. 
amygdala. Since no information was available for menopause or estrogen replacement treatment for the human female subjects, we interpreted these results as indicative of sexual dimorphism independently of age or estrogen status, although this will need to be tested in larger cohorts.

\section{Discussion}

We report sexually dimorphic gene expression profiles in the BA nucleus of the amygdala in human subjects. Changes at the level of single genes extend to homologous BLA nucleus in the mouse amygdala, albeit to a lesser extent. At the functional level, mitochondrial-related gene groups were consistently identified as the top biological pathways associated with male/female differences in both species. Together with prominent differences in other gene groups (Figure 2), our results suggest a sexual dimorphism in the expression level of an evolutionarily conserved pathway - circadian clock and mitochondrial function, potentially linked through sirtuin and nuclear hormone receptors.

In experimental models, circadian genes modulate energy metabolism by setting the periodic oscillations of expression and activity of enzymes to influence physiological and behavioral functions. Substantial evidence has shown that patients with major depression or bipolar disorder often display abnormalities in circadian rhythm, such as disturbances in the sleep/wake cycle and diurnal mood changes. Genetics studies have found associations with CLOCK, PER1-3, and CRY1, and Sirtuin genes in mood disorders [13-16]. In molecular/genetic studies, mitochondrial dysfunction has been associated with mood disorders $[17,18]$, and mood disorders are common in patients with mitochondrial disorders [19]. Thus, our findings suggest that baseline sexual dimorphism in mitochondrial density or functions may influence energy availability in the amygdala. Notably, while mitochondrial function was systemically identified as a top sex-biased gene family across species, the species-specific orthologous genes were not necessarily and systematically the same, but very close functionally (Figure $2 \mathrm{E}$ ). Because of identification of mitochondrial-related genes with closely related functions and locations across species, we speculate that the sex-biased mitochondria profile may be under conserved transcription control to display similar sexual dimorphism across species.

Limitations of this study are noted. The results will need to be confirmed at the protein and functional levels in independent and larger cohorts. Similarly, the potential contribution of race-specific amygdala gene expression will need to be assessed in larger cohorts. It is also unclear whether our findings in mid-life adults can be generalized to adolescent and elderly populations. Here we identified age effects in 2 of the 18 genes validated by
qPCR, suggesting that aging may not contribute to the evolutionary conserved genetic sex differences in amygdala. In addition, this study sampled 10 intact female mice and 12 female human subjects from 39-64 years old, which should considerably lower the impact of variable estrogen levels on our findings of genetic sex difference, although we cannot exclude residual effects.

In conclusion, we present evidence for significant male/ female differences in amygdala gene expression across species. Delineating the contribution of mitochondrial and circadian signaling in mood disorders will require additional work. For instance, sex-biased gene expression of circadian clock, nuclear hormone receptors, mitochondrial sirtuins and electron chain enzymes in an interlocking-feedback loop, may functionally translate into differential amygdala responsiveness (Figure 2E). This loop may lead to sex differences in metabolic capacity to meet energy demand, and might potentially contribute to the greater female vulnerability to mood disorders.

\section{Additional material}

\section{Additional file 1: Table S1. Sequence of human and mouse primers} used for qPCR measurements.

Additional file 2: Table S2. Sex-biased genes in human BA. Sex-biased genes detected by the microarray analysis in the postmortem basal amygdala samples from healthy human subjects.

Additional file 3: Table S3. Sex-biased genes in human LA.

Sex-biased genes detected by the microarray analysis in the postmortem lateral amygdala samples from healthy human subjects.

Additional file 4: Table S4. Sex-biased genes in mouse BLA Sex-biased genes detected by the microarray analysis in the basolateral amygdala from adult C57BL/6J mice.

Additional file 5: Table S5. Sex-biased genes in mouse LA. Sex-biased genes detected by the microarray analysis in the lateral amygdala from adult C57BL/6J mice.

\section{List of abbreviations used}

ACC: anterior cingulate cortex; AChE: acetylcholinesterase; BA: basal amygdala; BA9: Brodman area 9; BA47: Brodman area 47; BLA: basolateral amygdala; ETC: electron transport chain; IPA: Ingenuity Pathways Analysis; LA: lateral amygdala; qPCR: Real-time Quantitative PCR; RORB: retinoic acid orphan receptor $\beta$; SIRT: sirtuin; THRA: thyroid hormone receptor $a$.

\section{Acknowledgements}

We gratefully acknowledge support of this research from NIMH (MH077159, MH084060, MH085111), the University of Pittsburgh Brain Tissue Donation Program (MH084053), and Xingbin Wang for statistical support.

\section{Authors' contributions}

$L C L$ and ES designed this study. $L C L$ carried out the molecular experiments, performed the statistical analysis and drafted the manuscript. DAL provided technical and conceptual support for the human brain collection. The final manuscript was written by LCL and ES. All authors have read, edited and approved the final manuscript.

\section{Competing interests}

Dr. Lewis currently receives investigator-initiated research support from the BMS Foundation, Bristol-Myers Squibb, Curridium Ltd and Pfizer and in 20072010 served as a consultant in the areas of target identification and 
validation and new compound development to AstraZeneca, BioLine RX, Bristol-Myers Squibb, Hoffman-Roche, Lilly, Merck, Neurogen, and SK Life Science.

Received: 22 March 2011 Accepted: 4 May 2011 Published: 4 May 2011 doi:10.1186/1756-6606-4-18

Cite this article as: Lin et al: A human-mouse conserved sex bias in amygdala gene expression related to circadian clock and energy metabolism. Molecular Brain 2011 4:18.

\section{References}

1. Kendler KS: Gender differences in the genetic epidemiology of major depression. J Gend Specif Med 1998, 1(2):28-31.

2. Tondo L, Baldessarini RJ: Rapid cycling in women and men with bipolar manic-depressive disorders. The American journal of psychiatry 1998, 155(10):1434-1436.

3. Jazin $E$, Cahill $L$ : Sex differences in molecular neuroscience: from fruit flies to humans. Nature reviews 11(1):9-17.

4. Hines M, Allen LS, Gorski RA: Sex differences in subregions of the medial nucleus of the amygdala and the bed nucleus of the stria terminalis of the rat. Brain research 1992, 579(2):321-326.

5. Sheline $\mathrm{YI}$, Barch DM, Donnelly JM, Ollinger JM, Snyder AZ, Mintun MA: Increased amygdala response to masked emotional faces in depressed subjects resolves with antidepressant treatment: an fMRI study. Biological psychiatry 2001, 50(9):651-658.

6. Sibille E, Wang Y, Joeyen-Waldorf J, Gaiteri C, Surget A, Oh S, Belzung C, Tseng GC, Lewis DA: A molecular signature of depression in the amygdala. The American journal of psychiatry 2009, 166(9):1011-1024.

7. Svendsen CN, Bird ED: Acetylcholinesterase staining of the human amygdala. Neuroscience letters 1985, 54(2-3):313-318.

8. Kitt CA, Hohmann C, Coyle JT, Price DL: Cholinergic innervation of mouse forebrain structures. The Journal of comparative neurology 1994, 341(1):117-129.

9. The Ingenuity Pathways Knowledge Base. [http://www.ingenuity.com/]

10. Asher G, Gatfield D, Stratmann M, Reinke H, Dibner C, Kreppel F, Mostoslavsky R, Alt FW, Schibler U: SIRT1 regulates circadian clock gene expression through PER2 deacetylation. Cell 2008, 134(2):317-328.

11. Yang X, Downes M, Yu RT, Bookout AL, He W, Straume M, Mangelsdorf DJ, Evans RM: Nuclear receptor expression links the circadian clock to metabolism. Cell 2006, 126(4):801-810.

12. Galfalvy HC, Erraji-Benchekroun L, Smyrniotopoulos P, Pavlidis P, Ellis SP, Mann JJ, Sibille E, Arango V: Sex genes for genomic analysis in human brain: internal controls for comparison of probe level data extraction. BMC bioinformatics 2003, 4:37.

13. Roybal K, Theobold D, Graham A, DiNieri JA, Russo SJ, Krishnan V, Chakravarty S, Peevey J, Oehrlein N, Birnbaum S, Vitaterna MH, Orsulak P, Takahashi JS, Nestler EJ, Carlezon WA Jr, McClung CA: Mania-like behavior induced by disruption of CLOCK. Proceedings of the National Academy of Sciences of the United States of America 2007, 104(15):6406-6411.

14. Hampp G, Ripperger JA, Houben T, Schmutz I, Blex C, Perreau-Lenz S, Brunk I, Spanagel R, Ahnert-Hilger G, Meijer JH, Albrecht U: Regulation of monoamine oxidase A by circadian-clock components implies clock influence on mood. Curr Biol 2008, 18(9):678-683.

15. Soria V, Martinez-Amoros E, Escaramis G, Valero J, Perez-Egea R, Garcia C, Gutierrez-Zotes A, Puigdemont D, Bayes M, Crespo JM, Martorell L, Vilella E, Labad A, Vallejo J, Pérez V, Menchón JM, Estivill X, Gratacòs M, Urretavizcaya M: Differential association of circadian genes with mood disorders: CRY1 and NPAS2 are associated with unipolar major depression and CLOCK and VIP with bipolar disorder. Neuropsychopharmacology 35(6):1279-1289.

16. Abe N, Uchida S, Otsuki K, Hobara T, Yamagata H, Higuchi F, Shibata T, Watanabe $Y$ : Altered sirtuin deacetylase gene expression in patients with a mood disorder. Journal of psychiatric research.

17. Konradi C, Eaton M, MacDonald ML, Walsh J, Benes FM, Heckers S: Molecular evidence for mitochondrial dysfunction in bipolar disorder. Archives of general psychiatry 2004, 61(3):300-308.

18. Rollins B, Martin MV, Sequeira PA, Moon EA, Morgan LZ, Watson SJ, Schatzberg A, Akil H, Myers RM, Jones EG, Wallace DC, Bunney WE, Vawter MP: Mitochondrial variants in schizophrenia, bipolar disorder, and major depressive disorder. PloS one 2009, 4(3):e4913.

19. Fattal O, Link J, Quinn K, Cohen BH, Franco K: Psychiatric comorbidity in 36 adults with mitochondrial cytopathies. CNS spectrums 2007, 12(6):429-438

\section{Submit your next manuscript to BioMed Central and take full advantage of:}

- Convenient online submission

- Thorough peer review

- No space constraints or color figure charges

- Immediate publication on acceptance

- Inclusion in PubMed, CAS, Scopus and Google Scholar

- Research which is freely available for redistribution 